\title{
Isolation and Functional Characterization of a B3 Transcription Factor Gene FUSCA3 Involved in Pre-Harvest Sprouting Resistance in Wheat
}

\author{
Xinguo Wang1,2*, Lili Liu1 ${ }^{*}$, Yanli Wang1, Xiaodan Meng1, Yumei Jiang1,2, Yongchun Li1,2, Lei Li1,2\#, \\ Jiangping Ren ${ }^{1,2 \#}$
}

${ }^{1}$ College of Agronomy, Agricultural University of Henan, Zhengzhou, China

${ }^{2}$ National Engineering Research Center for Wheat, Zhengzhou, China

Email: "nercw@163.com, "xmzxrjp@126.com

How to cite this paper: Wang, X.G., Liu, L.L., Wang, Y.L., Meng, X.D., Jiang, Y.M., Li, Y.C., Li, L. and Ren, J.P. (2021) Isolation and Functional Characterization of a B3 Transcription Factor Gene FUSCA3 Involved in Pre-Harvest Sprouting Resistance in Wheat. Agricultural Sciences, 12, 844-862. https://doi.org/10.4236/as.2021.128054

Received: July 12, 2021

Accepted: August 8, 2021

Published: August 11, 2021

Copyright $\odot 2021$ by author(s) and Scientific Research Publishing Inc. This work is licensed under the Creative Commons Attribution International License (CC BY 4.0).

http://creativecommons.org/licenses/by/4.0/

(c) (i) Open Access

\begin{abstract}
Pre-harvest sprouting (PHS) reduces yields and grain quality, resulting in seriously economic losses in wheat. It has been showed that PHS is significantly correlated to seed dormancy levels. FUSCA3 (FUS3) gene is considered to be the key regulator of seed dormancy. However, little information is available about the function of FUS3 gene (TaFUS3) in wheat. In this study, three homologous genes were identified in wheat grain, and their functions were investigated by gene silencing. Three full-length DNA (3477, 3534 and $3501 \mathrm{bp}$ ) and cDNA (1015, 1012 and $1015 \mathrm{bp})$ sequences encoding a B3 transcription factor, designated TaFUS3-3A, TaFUS3-3B and TaFUS3-3D, were first isolated from common wheat. The transcription of three TaFUS3 genes in seed development and germination process was detected. TaFUS3-3B and TaFUS3-3D had similar expression profiles, and high levels of gene transcripts were detected in seeds at $25 \mathrm{DAP}$ (days after pollination) and after $24 \mathrm{~h}$ of imbibition. However, the transcription of TaFUS3-3A was not detected. Silencing of TaFUS3 in common wheat spikes resulted in increased seed germination and PHS. Compared with wild-type, the TaFUS3-silenced plants showed increased expression of genes related to GA biosynthesis and ABA metabolism, and decreased expression of genes associated with ABA biosynthesis. Moreover, silencing of TaFUS3 in wheat plants led to a decrease in embryo sensitivity to $\mathrm{ABA}$ and changed the expression of genes involved in ABA signal transduction. The results of gene silencing indicated that TaFUS3 plays a positive role in wheat seed dormancy and PHS-resistance, which might be associated with ABA, GA level and signal transduction.
\end{abstract}




\section{Keywords}

Wheat (Triticum aestivum L.), FUSCA3, Molecular Cloning, Virus-Induced Gene Silencing (VIGS), Pre-Harvest Sprouting

\section{Introduction}

Pre-harvest sprouting (PHS) denotes grains germinating on the mother plants when they are subjected to the rainy and humid environment prior to harvest [1]. PHS of wheat grains not only causes the decline in grain yield, but also affects the end-use quality of the grain, resulting in severe economic losses [2] [3]. PHS is a worldwide problem. In some wheat-growing countries, such as the United States, Canada, Australia, and Japan, the problems associated with PHS occur frequently [4] [5] [6] [7]. In China, heavy PHS sometimes occurs on $>83 \%$ of the wheat acreage [8]. Therefore, increasing resistance to PHS is an important current objective in improving wheat varieties.

Wheat PHS is a complex quantitative trait regulated by multiple genes [9]. Many studies have shown that seed dormancy level is one of the main factors determining the resistance to PHS in wheat [2] [10]. Seed dormancy is finely controlled by the seed maturation program, and ABA plays a key role in regulating seed dormancy [11] [12]. Genetic and molecular studies have shown that many of the genes participating in seed dormancy are known to be involved in ABA synthesis and signal transduction [10]. Studies in Arabidopsis showed that seed dormancy and germination were controlled by at least four major regulators, namely ABA INSENSITIVE3 (ABI3), FUSCA3 (FUS3), LEAFY COTYLEDON2 (LEC2), and LEAFY COTYLEDON 1 (LEC1) [13] [14] [15]. ABI3, FUS3 and $L E C 2$ are plant-specific genes, encoding transcription factor of the $\mathrm{B} 3$ domain family, whereas $L E C 1$ encodes a HAP3 subunit of the CCAAT binding factor [16] [17] [18] [19]. The loss-of-function mutants of these genes showed reduced accumulation of seed storage proteins and enhanced precocious germination of immature embryos [20] [21] [22]. Although these genes have similar functions, their expression patterns differ during seed development [23] [24]. LEC1 and $L E C 2$ are expressed during the first period of embryonic development, followed by FUS 3 and finally by $A B I 3$; all genes are active in the embryo, and $L E C 2$ and FUS3 are active also in the endosperm [17] [19]. Moreover, the spatio-temporal expressions of $L E C 2 / A B I 3 / F U S 3 / L E C 1$ are transcriptionally cross-regulated and act together to control seed maturation and dormancy [25].

Recently, it has been demonstrated that FUS3 is a crucial molecular switch in regulating a transition from seed dormancy to germination [26] [27]. FUS3 usually acts as both activator and repressor of seed germination and dormancy by interacting with hormone signaling and synthesis pathways [20] [28]. It has been reported that FUS3 accumulates preferentially in the epidermis, vasculature and radicle tips of embryos. FUS3 expression is regulated by auxin [26]. The 
C-terminal domain (CTD) of FUS3 is responsible for instability of the FUS3 protein and is involved in hormone sensitivity [29]. During late seed maturation, FUS3 can promote dormancy and inhibit precocious germination of immature seeds by increasing $\mathrm{ABA}$ concentration and repressing GA biosynthesis genes. In addition, FUS3 protein is regulated by ABA and GA. The stability and concentration of the FUS3 are regulated negatively by ABA and positively by GA [26] [29] [30]. Similarly, during seed imbibition, FUS3 protein acts as a seed germination repressor through regulating ABA/GA ratio [31]. Furthermore, over-expression of Arabidopsis FUS3 increases the sensitivity to high temperature and delays seed germination due to a high ABA level [32] [33]. These results indicated that FUS3 plays a critical role in inducing dormancy and inhibiting germination of seed by regulating $\mathrm{ABA}$ and $\mathrm{GA}$ levels.

Wheat is a globally important crop, accounting for $20 \%$ of the calories consumed by humans. Research that focuses on mechanisms of seed dormancy regulation at the molecular level is very important for accelerating the process of improving wheat varieties. Although the functions of FUS3 gene have been studied extensively in Arabidopsis, and putative orthologs have been described in several monocot species [34] [35], FUS3 gene has not experimentally been proven to have a role in PHS tolerance in wheat, and wheat homologues for FUS3 have not been identified. Therefore, in this study, we isolated the sequences of $\mathrm{Ta}$ FUS3 homoeologs in hexaploid wheat, analyzed the effect of silencing the FUS3 gene using the barley stripe mosaic virus (BSMV)-mediated virus-induced gene silencing (VIGS) technique. These research data will provide fundamental insight for future studies regarding the functions of FUS3 gene in seed dormancy and PHS.

\section{Materials and Methods}

\subsection{Plant Materials and Growth Conditions}

Wheat cultivar (Triticum aestivum L.) Huaimai 0360 (highly PHS-resistant cultivar), was used for this study. For gene cloning and expression experiments, 100 surface-sterilized seeds were germinated on moist filter paper in growth chambers at $25^{\circ} \mathrm{C}$ under 12 -h light/12-h dark conditions and transplanted into pots in a naturally lit glasshouse with standard irrigation and fertilization until mature. During this period, developing seeds were collected at 15,20,25, and 30 days after pollination (DAP). For germination, mature seeds were surface-sterilized and then imbibed on water moistened filter paper in Petri dishes in a temperature-controlled cultivation chamber (darkness at $25^{\circ} \mathrm{C}$ ). 3 - 5 seeds were collected at $0,6,12,24,36$, and $48 \mathrm{~h}$ after imbibition commenced. All the collected plant samples were snap-frozen in liquid nitrogen immediately after harvesting and stored at $-80^{\circ} \mathrm{C}$ until use. Each treatment was repeated thrice.

For VIGS experiments, wheat cultivar Huaimai 0360 was grown in the agricultural and experimental field of Henan Agricultural University (Zhengzhou, China) during the wheat-growing season. The plot area was $15 \mathrm{~m}^{2}$ (length $5.0 \mathrm{~m}$ 
$\times$ width $3.0 \mathrm{~m}$ ) and was watered and fertilized as needed throughout the growth period.

At anthesis, an arch shed (length $5.0 \mathrm{~m} \times$ width $3.0 \mathrm{~m} \times$ height $1.5 \mathrm{~m}$ ) was built directly above the wheat plants according to the method described by Liu et al. [36]. One hundred wheat spikes in the same heading stage and of similar size were labeled. During 1 day before and after BSMV inoculation, the PVC plastic film was covered with soil to prevent water loss from affecting the spread of the virus. Developing grains in the middle of BSMV-infected spikes were collected at $21,28,35$, and 45 days post inoculation (dpi) at 10:00 a.m., snap-frozen in liquid nitrogen and then stored at $-80^{\circ} \mathrm{C}$ for transcription analysis. At maturity, spikes of BSMV-infected wheat plants were harvested and stored at room temperature until use. Uniform spikes at the anthesis stage were used for BSMV-VIGS inoculation.

\subsection{Isolation of the Wheat FUS3 Homologues}

Genomic DNA was isolated from seed at 15 30 DAP using the CTAB method. Gene specific primers TaFUS3-F (5'-TCC TCC GCC TTG ACC TCC T-3') and TaFUS3-R (5'-CAA GGC TGG TGA CTC TGA ACT-3') were designed based on the sequence of TaFUS3 [37] using Primer 5.0 software. The PCR was performed in an Applied Biosystems-2720 thermal cycler in total volumes of $50 \mu \mathrm{L}$, including $5 \mu \mathrm{L}$ of $10 \times$ PCR buffer, $100 \mathrm{mM} \mathrm{dNTP}, 0.4 \mu \mathrm{M}$ of each primer, 2.5 unit of Taq DNA polymerase (TIANGEN, Beijing), and $100 \mathrm{ng}$ of template DNA. The PCR cycling conditions comprised an initial cycle at $95^{\circ} \mathrm{C}$ for $3 \mathrm{~min}$ followed by 35 cycles of $94^{\circ} \mathrm{C}$ for $30 \mathrm{~s}$, annealing at $58^{\circ} \mathrm{C}$ for $1 \mathrm{~min}$ and $72^{\circ} \mathrm{C}$ for $2 \mathrm{~min}$, with a final extension at $72^{\circ} \mathrm{C}$ for $10 \mathrm{~min}$. PCR products were separated by electrophoresis in $1.0 \%$ agarose gels. Targeted fragments of expected size were recovered and cloned into the $\mathrm{pMD} 18$ - $\mathrm{T}$ vector. Selected 20 positive clones were sequenced by Henan Shangya Technology Co., Ltd. Sequence alignment was performed by using DNAMAN software. Chromosome locations of cloned sequences were identified at urgi (https://urgi.versailles.inra.fr/blast/blast.php).

\subsection{Functional Analysis by BSMV-VIGS}

For VIGS experiments, a $\gamma$ RNA-based BSMV vector was utilized to silence FUS3 gene in wheat as described previously [38]. Since wheat is hexaploid, there are usually three copies of genes. To make TaFUS3 gene silenced completely, a 247 bp cDNA fragment from the wheat FUS3 gene that contains Nhe I sites was amplified with specific primers designed in the conserved regions of the three copies by Primer 5.0 software. The forward and reverse primers were 5'-GCG GGA GAT GAT CTA GTT GC-3' and 5'-AAT CAC TGA ATG GGT CGA AGA-3', respectively. Then, the amplified PCR products and the BSMV- $\gamma$ empty vector were individually digested with restriction enzyme $N$ he I. The digestion results were verified by agarose gel electrophoresis. The correct target fragments were ligated into the BSMV- $\gamma$ empty vector and sequenced. The inserted clone in re- 
verse was selected as the recombinant vector BSMV: TaFUS3 and subsequently used for gene silencing. For identifying wheat plants with the VIGS response, the BSMV: GFP was used as an infection control.

In vitro transcription of viral RNAs was carried out as described by Feng et al. [38]. The plasmids of BSMV- $\alpha$, BSMV- $\beta$, BSMV- $\gamma$-derivative clones (BSMV-TaFUS3 and BSMV-GFP) were digested with restriction enzymes $M l u$ I and Spe I. Then, the linearized products were examined by electrophoresis. The correct linearized products were purified, and transcription was performed using a RiboMAX ${ }^{\mathrm{TM}}$ Large Scale RNA Production Systems-T7 kit (Promega, USA) following the manufacturer's protocol. Transcripts of each of the BSMV plasmids were mixed in a 1:1:1 ratio and 22.5 volumes of FES buffer [39] were added to the transcript mixture. This mixture was then applied to the young wheat spikes by rub inoculation. $15 \mu \mathrm{L}$ of BSMV: TaFUS3 or BSMV: GFP transcript mixture was used for each spike. The details of the BSMV inoculation and wheat plant growth conditions were described by Liu et al. [36].

\subsection{Seed Germination and Spike Sprouting Test}

180 wheat seeds from both the BSMV-TaFUS3 and BSMV-GFP plants (40 dpi) with uniform size and plumpness were surface sterilized in $70 \%$ alcohol for 1 min, and then in $0.1 \% \mathrm{HgCl}_{2}$ for $5-10 \mathrm{~min}$ and washed four times with sterile distilled water. Six groups (30 grains/group) were distributed on two layers of filter paper in a $9-\mathrm{cm}$ Petri dish containing $10 \mathrm{~mL}$ of sterilized water, and germinated at $25^{\circ} \mathrm{C}$ under continuous dark for 7 days. Three of them were used to investigate the germination rate and the other three groups were used for the expression analysis of ABA/GA synthetic and metabolic genes. The number of germinated seeds (radicle protruding through the seed coat) was recorded daily. Germination ratio refers to the number of germinated seed as a proportion of the total number of seeds. For the gene expression analysis, germinated seeds were harvested at $24,48,72$, and $96 \mathrm{~h}$ snap-frozen in liquid nitrogen and stored at $-80^{\circ} \mathrm{C}$ for further analysis.

Spike sprouting tests were performed with intact spikes (40 and $45 \mathrm{dpi}$ ). Twelve spikes each from the BSMV-TaFUS3 and BSMV-GFP plant groups were surface sterilized in $0.1 \% \mathrm{HgCl}_{2}$ for $10 \mathrm{~min}$, and washed four times with sterilized water, vertically inserted into a foam board (length $20 \mathrm{~cm} \times$ width $20 \mathrm{~cm} \times$ height $3 \mathrm{~cm}$ ), and covered by a plastic film to prevent water loss. Water was sprayed once every $6 \mathrm{~h}$. After 7 days, the test spikes were taken out and immediately threshed manually to record seed germination. The calculation method of germination rate was the same as for the seed germination test. In addition, the plumule and radicle length was measured on germinated seeds. All tests were performed in a climate chamber at $25^{\circ} \mathrm{C}(24 \mathrm{~h}$ darkness $)$.

\subsection{ABA Treatment}

Seeds from BSMV-TaFUS3 and BSMV-GFP plants (40 dpi) were soaked in steri- 
lized water for $4 \mathrm{~h}$, and then the embryos were isolated with dissecting needles under aseptic conditions and were placed on two layers of filter paper in a $9 \mathrm{~cm}$ Petri dish containing $10 \mathrm{~mL}$ of sterilized water with $50 \mu \mathrm{M} \mathrm{ABA}$ at $25^{\circ} \mathrm{C}$ in darkness for $48 \mathrm{~h}$. RNA was extracted from embryos after 0 and $48 \mathrm{~h}$ of treatment. The experiments were performed in triplicate.

\subsection{Quantitative Real-Time PCR (qRT-PCR)}

The RNA extraction and the first strand cDNA synthesis were described in our previous study [40]. Based on the sequences of the genes, including TaFUS3-3A, TaFUS3-3B, TaFUS3-3D, TaNCED1, TaNCED2, TaCYP707A2, TaGA20ox-D4, TaGA20ox1d, TaABI3, TaABI4, TaABI5, TaPKABA1, and $\beta$-actin, specific primers were designed using Primer 5.0 software. The primer sequences and amplified product are provided in Table 1. The qRT-PCR analysis was performed with a SYBR Green quantitative RT-PCR kit (TaKaRa, Dalian, China) on a CFX connect real-time system (Bio-RAD, USA) following the manufacturer's instructions. The expression level of each gene was determined using the comparative $\mathrm{Ct}$ method. Relative quantification for each gene was calculated by the $2^{-\Delta \Delta \mathrm{Ct}}$ method using wheat $\beta$-actin gene as an internal control [41].

\section{Results}

\subsection{Isolation and Characterization of TaFUS3 Homologues in Wheat}

Based on the known cDNA sequence of TaFUS3 gene, a pair of specific primers

Table 1. Primer sequences for gene expression assay.

\begin{tabular}{|c|c|c|c|c|}
\hline \multirow{2}{*}{ Gene } & \multirow{2}{*}{ GenBank No. } & \multicolumn{2}{|c|}{ Primer sequence $\left(5^{\prime}-3^{\prime}\right)$} & \multirow{2}{*}{ Amplicon length (bp) } \\
\hline & & Forward & Reverse & \\
\hline TaFUS3-3A & MZ408248 & GTGGCTGGGTTGCGAGTT & TGTTCGGCCAGTATCTGTTCC & 196 \\
\hline TaFUS3-3B & MZ408249 & GCCGAACAACAAGAGCAGG & GTGGTGGGATTAGAGACACATACTT & 239 \\
\hline TaFUS3-3D & MZ408250 & GTGGCTGGGTTGCGAGTTA & GTGCATTTAGCAAATCATGCGTA & 160 \\
\hline TaFUS3-VIGS & - & CGAGATGTTTGATGGGATTTT & GGTTGGGAAACAAAGAAAGC & 188 \\
\hline TaNCED1 & JQ772528 & GTCGGAGATGATGTGGGTG & CCGTGTCGTTGAAGATGGA & 137 \\
\hline TaNCED2 & LC077862 & GTCGGAGATGGTGTGGGT & TCGTTGAAGATGGAGTCGG & 132 \\
\hline ТаСУР707A2 & AB849504 & CTGCCCCCTGGCTCCAT & GCCGTACCGCTTCTGCTT & 102 \\
\hline TaGA20ox-D4 & LN828669 & GCGGCAGCAAAAACAAAT & GTCGACCACAGGCACGTC & 94 \\
\hline TaGA20ox1d & FR716527 & TCGCTGGAGATCATGGAG & GCACGGCGGGTAGTAGTT & 114 \\
\hline TaABI3 & DQ517494 & CTGGTGACTTTGTTCGGTCC & TGGCATTCTTGTGCTTGG & 138 \\
\hline TaABI4 & AY781355 & GGATGCTGCCCGTGCTTAT & TGAGTGGTTGGCTGATGTTGTAG & 178 \\
\hline TaABI5 & KX002276 & TCCTGTGGTGGGTGCTGG & GCTGCTGTGAGGGTTGTGC & 188 \\
\hline TaPKABA1 & DQ343302 & CCCTGATGAGCCAAGGAACT & CGGGACAGTAGATGTACGCAGT & 111 \\
\hline$\beta$-actin & AB181991 & TTTGAAGAGTCGGTGAAGGG & TTTCATACAGCAGGCAAGCA & 196 \\
\hline
\end{tabular}


TaFUS3-F and TaFUS3-R were designed in the 5' and 3' non-coding regions, respectively, to amplify the complete gene DNA sequence from wheat cultivar "Huaimai 0360". An amplicon of about 3500 bp was obtained. After sequencing, three genes, containing seven exons and six introns, and with sequence lengths of 3477,3534 and, 3501 bp was obtained (Figure 1). The URGI BLAST analysis showed that the three sequences were highly similar to the, respectively, $3 \mathrm{~A}, 3 \mathrm{~B}$ and $3 \mathrm{D}$ sequences from the long arm of chromosome 3 in Chinese spring. So, the three TaFUS3 homologues were named TaFUS3-3A (GenBank accession MZ408248), TaFUS3-3B (GenBank accession MZ408249) and TaFUS3-3D (GenBank accession MZ408250), respectively. The corresponding cDNA sequences of TaFUS3-3A, TaFUS3-3B and TaFUS3-3D were 1015, 1012 and 1015 bp in length, and contained 906, 909 and 906 bp open reading frames (ORF), respectively. TaFUS3-3A, TaFUS3-3B and TaFUS3-3D were predicted to encode proteins containing 301, 302 and 301 amino acid, respectively, and shared $98.57 \%$ similarity.

\subsection{Transcription Profiling of the TaFUS3 Homoeologs during Seed Development and Germination in Wheat}

The expression profiles of three TaFUS3 genes in seed development and germination stages were investigated by qRT-PCR using gene-specific primer pairs TaFUS3-3A-F/R, TaFUS3-3B-F/R and TaFUS3-3D-F/R (Table 1). Expression analysis indicated that the significant differences in transcription were observed among the three homoeologous genes. TaFUS3-3B and TaFUS3-3D had similar expression patterns (Figure 2), whereas the expression of TaFUS3-3A was almost undetectable in all samples (data not shown). During grain filling stage, the transcription of TaFUS3-3B and TaFUS3-3D showed a gradual upward trend at 15 - 25 DAP, and then declined quickly at 30 DAP (Figure 2(a)). During seed imbibition, the expression of TaFUS3-3B and TaFUS3-3D showed an up-regulation trend in the $0-24 \mathrm{~h}$ period. Afterwards ( $36-48 \mathrm{~h}$ ), the transcripts of the two genes showed gradual declined (Figure 2(b)). Additionally, the two TaFUS3 genes also shared different transcript abundances during seed development and germination process. For instance, TaFUS3-3D had higher expression level than

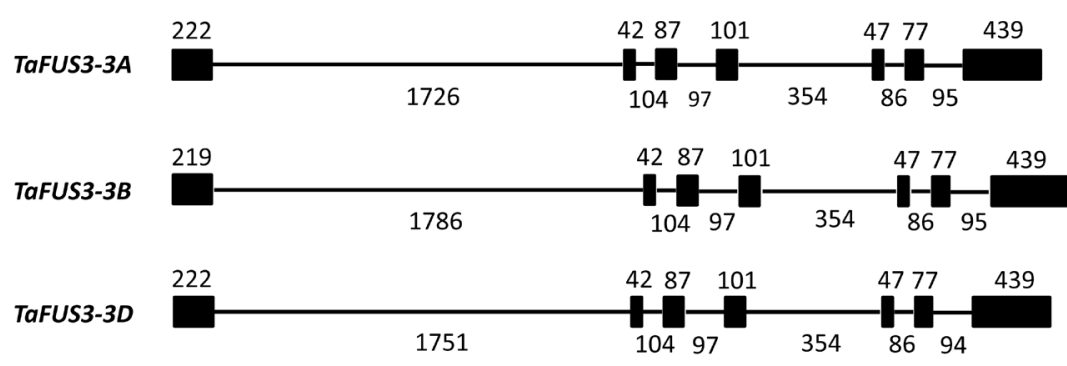

Figure 1. Schematic comparison of the TaFUS3 homologs genes. Black boxes indicate exons and solid lines indicate introns. The numbers above the lines and below the boxes indicate the size of exons and introns in bp, respectively. 


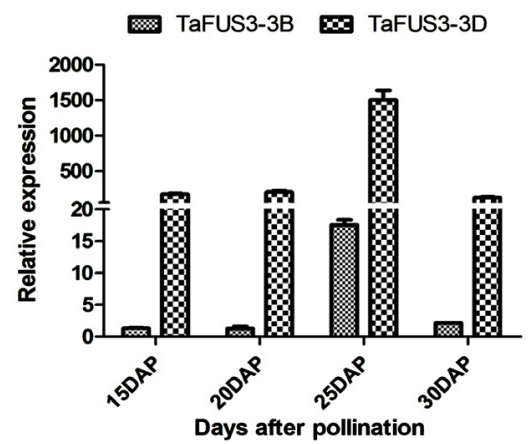

(a)



(b)

Figure 2. Expression profiles of the TaFUS3 genes determined by quantitative real-time PCR Analysis. ((a) and (b)) The expression levels of TaFUS3-3B and TaFUS3-3D in seed development (a) and germination (b) in wheat cultivar Huaimai 0360.

that of TaFUS3-3B. These results indicated that the two TaFUS3 homoeologs might play an important role in wheat seed development and germination.

\subsection{Molecular Identification of the Barley Stripe Mosaic Virus-Wheat FUSCA3 (BSMV-TaFUS3) Infected Wheat Plants}

To functionally test the role of TaFUS3 gene, we used a virus-induced gene silencing (VIGS) approach in the cultivar "Huaimai 0360". This cultivar has a relatively high seed dormancy level. A 247-bp cDNA fragment of the TaFUS3 gene was isolated. Then, a recombinant viral vector BSMV: TaFUS3 was constructed and used to inoculate the wheat spikes during the heading stage under field conditions. We collected developing seeds at 21, 28, 35, and $45 \mathrm{dpi}$ and then mature seeds (45 dpi) were imbibed for $24,48,72$, and $96 \mathrm{~h}$. The qRT-PCR analysis showed that the transcript level of TaFUS3 in the BSMV: TaFUS3-silenced plants was significantly decreased (by $61 \%, 41 \%, 55 \%$, and $25 \%$ at $21,28,35$, and 45 dpi, respectively) compared with the control plants (Figure 3(a)). During seed germination, the transcript abundance of TaFUS3 declined by $18 \%-24 \%$ in mature seeds (45 dpi) imbibed for 48 - $96 \mathrm{~h}$ (Figure 3(b)). These findings indicated the target gene was successfully repressed in BSMV: TaFUS3-silenced plants.

\subsection{Silencing of TaFUS3 Increased Seed Germination and Spike Sprouting Capacities}

The effect of TaFUS3 knockdown on the seed germination was characterized at $40 \mathrm{dpi}$. The seed germination capacity of BSMV-TaFUS3-silenced plants was always higher than that of control plants (BSMV: GFP infected) for 7 days of imbibition (Figure 4(a)). Moreover, the germination speed was faster in BSMV:TaFUS3 than BSMV:GFP infected plants following the addition of water. As shown in Figure 4(b), the seed germination rate in TaFUS3-silenced plants reached $16.7 \%, 24.5 \%$, and $27.8 \%$ on the first, second and third day of germination, which were 5 times, 3.7 times and 2.8 times of the control, respectively. 


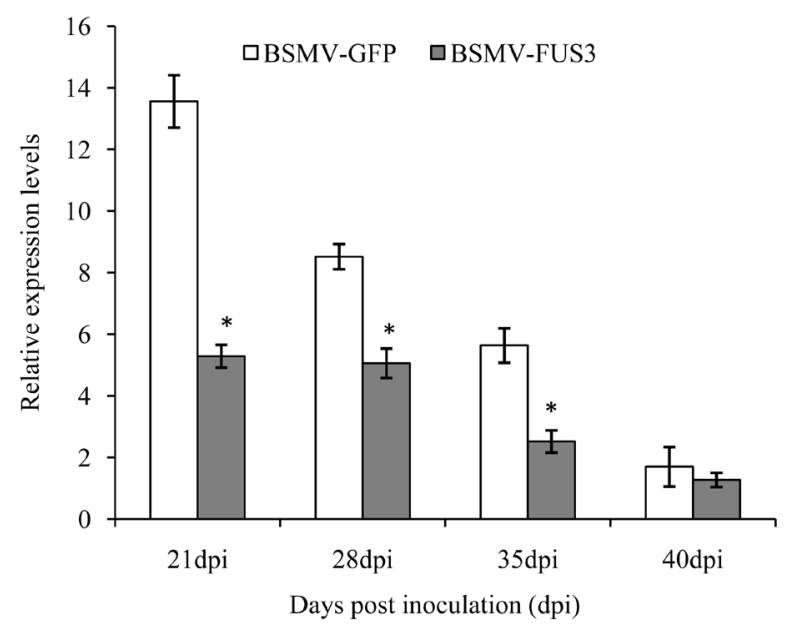

(a)

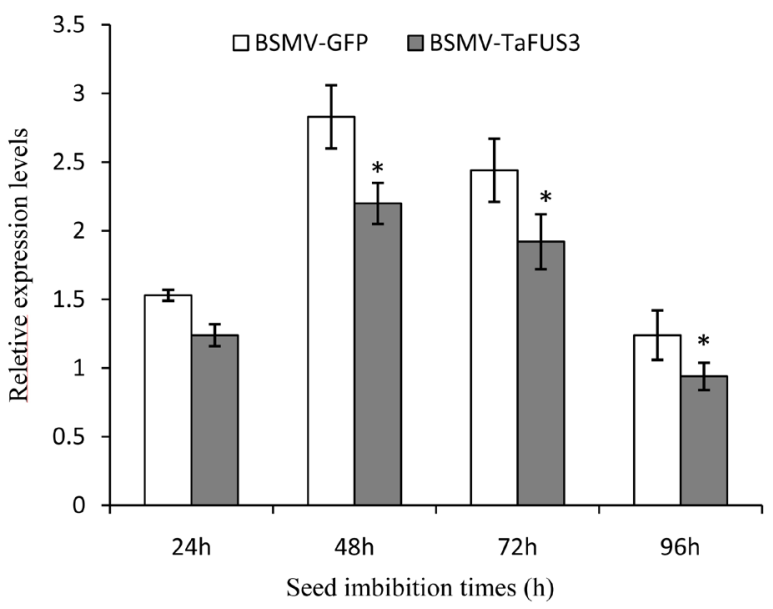

(b)

Figure 3. Identification of the BSMV-VIGS wheat plants and expression profiles of the TaFUS3 genes determined by quantitative real-time PCR Analysis. ((a) and (b)) Relative expression level of the TaFUS3 gene during seed development (a) and germination (b) in the BSMV-TaFUS3-inoculated and the BSMV-GFP-inoculated plants. Asterisks indicate significant differences $(p<0.05)$.

Similar results in PHS experiment were observed at 40 and 45 dpi. The data showed that the sprouting capacity was obviously higher in spikes of BSMV:TaFUS3 than BSMV:GFP plants (Figure 4(c)). Compared with the control, the germination rates in seed of BSMV-TaFUS3 wheat spike increased on average 50\% (40 dpi) and 24.1\% (45 dpi) (Figure 4(d)). In addition, the plumule and radicle lengths in grains of BSMV: TaFUS3 wheat spike were increased by, respectively, $60.4 \%$ and $55.8 \%$ at $40 \mathrm{dpi}$ (Figure $4(\mathrm{e})$ ) and $14.3 \%$ and $32.5 \%$ at $45 \mathrm{dpi}$ (Figure 4(f)). These findings indicated that the sprouting speed was significantly faster in spikes of BSMV: TaFUS3 than BSMV-GFP wheat. Above results suggested that TaFUS3 silencing resulted in enhanced seed germination and PHS in wheat grains; thus, TaFUS3 may act as a negative regulator of wheat seed germination. 


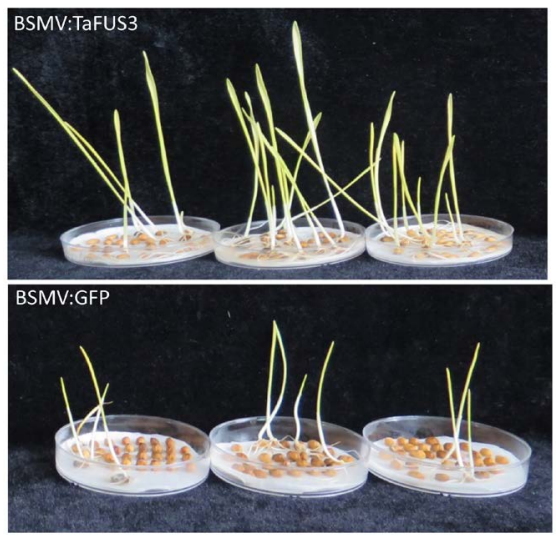

(a)
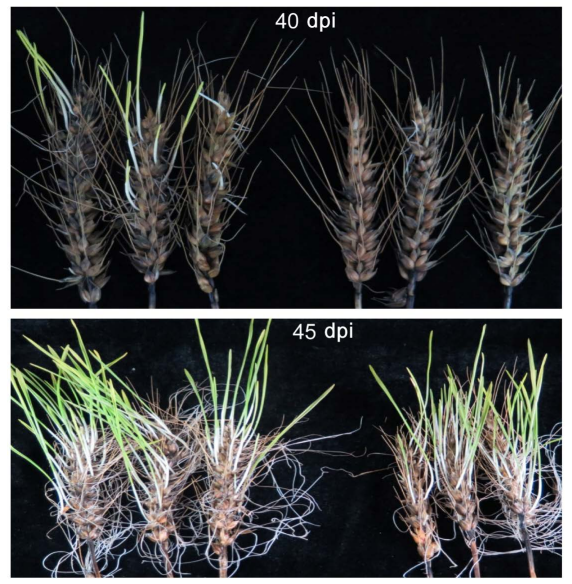

BSMV-TaFUS3

(c)

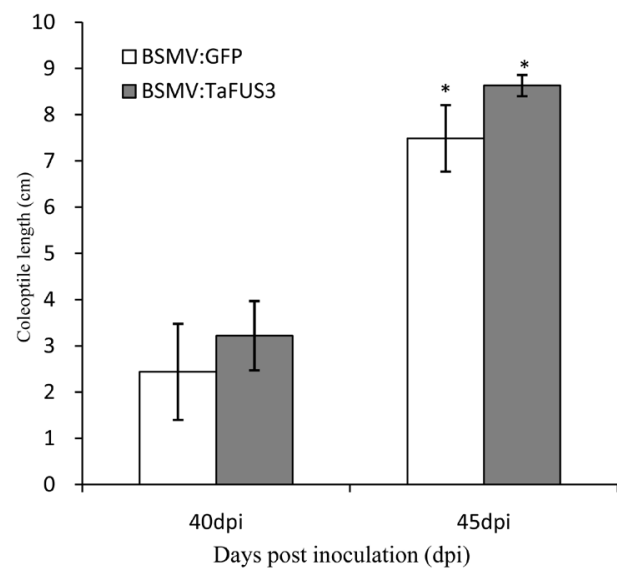

(e)

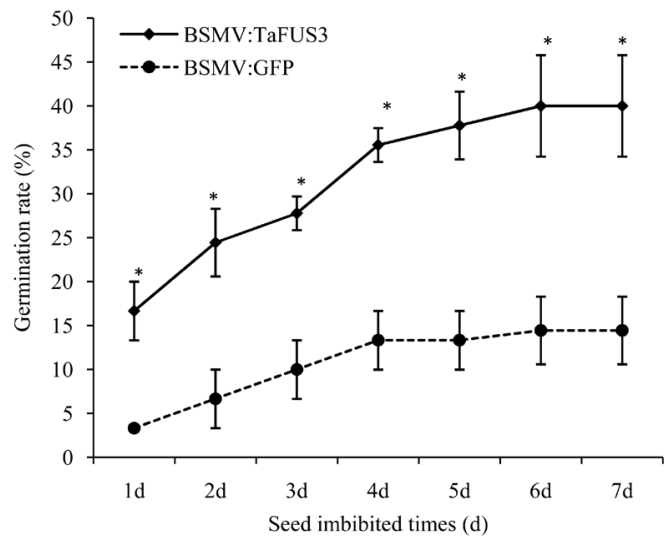

(b)

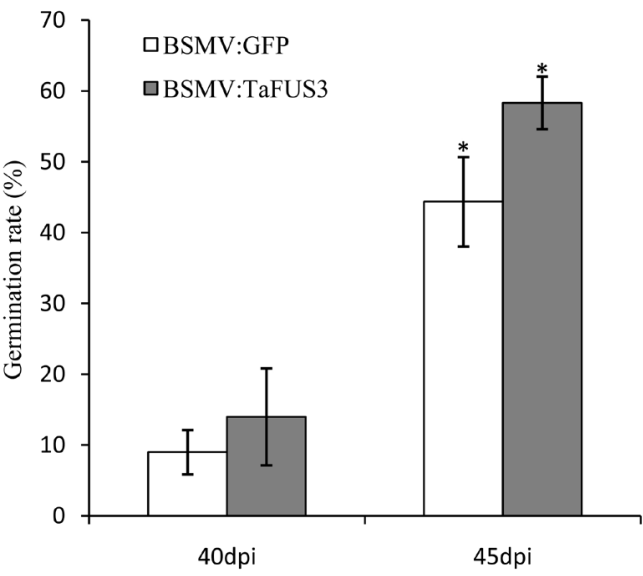

Days post innoculation (dpi)

(d)

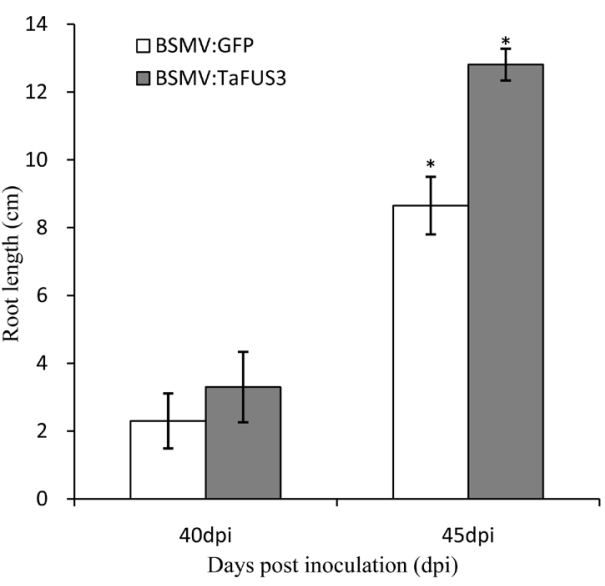

(f)

Figure 4. Characterization of seed germination and PHS from the BSMV-TaFUS3-infected and BSMV-GFP-infected wheat plant. ((a) and (b)) Seed germination phenotypes and germination rate of BSMV-TaFUS3-infected and BSMV-GFP-infected wheat plants at $40 \mathrm{dpi}$ incubated on moist filter paper for $7 \mathrm{~d}$. ((c) and (d)) Spike sprouting phenotypes and germination rate of BSMV-TaFUS3-infected and BSMV-GFP-infected plants incubated for $7 \mathrm{~d}$ under misting conditions at 40 and 45 dpi. ((e) and (f)) Coleoptile and radicle lengths of the BSMV-TaFUS3-infected and BSMV-GFP-infected wheat spikes after $7 \mathrm{~d}$ under misting conditions at 40 and $45 \mathrm{dpi}$. 


\subsection{Silencing of TaFUS3 Changed the Transcript Levels of Genes Related to GA/ABA Biosynthesis and ABA Metabolism during Seed Germination}

To define the role of TaFUS3 in regulating seed germination and PHS, qRT-PCR analyses were carried out to detect the expression levels of ABA biosynthesis genes TaNCED1, TaNCED2, GA biosynthesis genes TaGA20ox-D4, TaGA20ox1d and ABA metabolism gene TaCYP707A2 (Table 1). The result revealed that the transcription of one GA synthesis genes (TaGA20ox1d) and one ABA metabolism gene (TaCYP707A2) was evidently induced and their transcript levels in TaFUS3-silenced plants were up-regulated on average by 1.9 and 2.0 times, respectively, in the seeds imbibed for 48 hours (Figure 5). However, the expression of ABA biosynthesis genes TaNCED1 and TaNCED2 was obviously inhibited and the transcription levels of TaNCED2 in TaFUS3-silenced plants decreased by $32.86 \%$ (Figure 5), indicating that TaFUS3 might control seed dormancy and PHS -resistance by positively regulating ABA synthesis genes and negatively regulating $\mathrm{ABA}$ metabolism and GA synthesis genes.

\subsection{Decreased Expression of TaFUS3 Gene in Wheat Granted Reduced ABA Sensitivity through Negatively or Positively Regulating Different Genes Involved in the ABA Signaling Pathway}

The expression levels of TaFUS3 and endogenous $A B I 3, A B I 4, A B I 5$, and PKABA1 (Table 1) were analyzed in embryos of the BSMV-infected plants upon ABA treatment. As shown in Figure 6, after ABA treatment, the expression of these genes was induced. Compared with the control, the relative transcript level of TaFUS3 in BSMV:TaFUS3 plants was significantly lower. In addition, the expression of TaFUS3 in TaFUS3-silenced plants significantly up-regulated the expression of $P K A B A 1$ and markedly down-regulated the expression of $A B I 5$

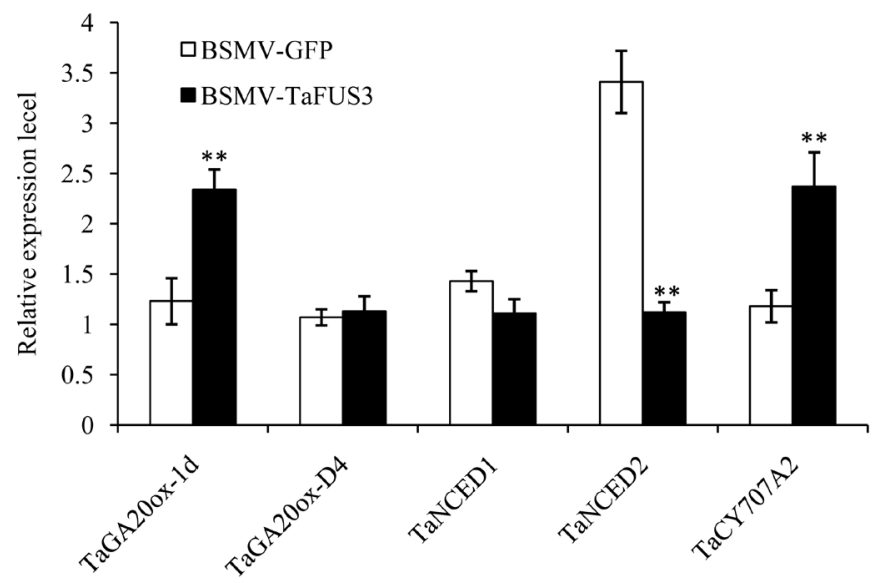

Figure 5. Expression profiles of TaGA20ox1d, TaGA20ox-D4, TaNCED1, TaNCED2, and TaCYP707A2 genes determined by qRT-PCR analysis in the process of seed germination. Each value is the mean \pm standard deviation of three independent biological replicates. " $* *$ " indicate significant differences at $p<0.01$ level. 


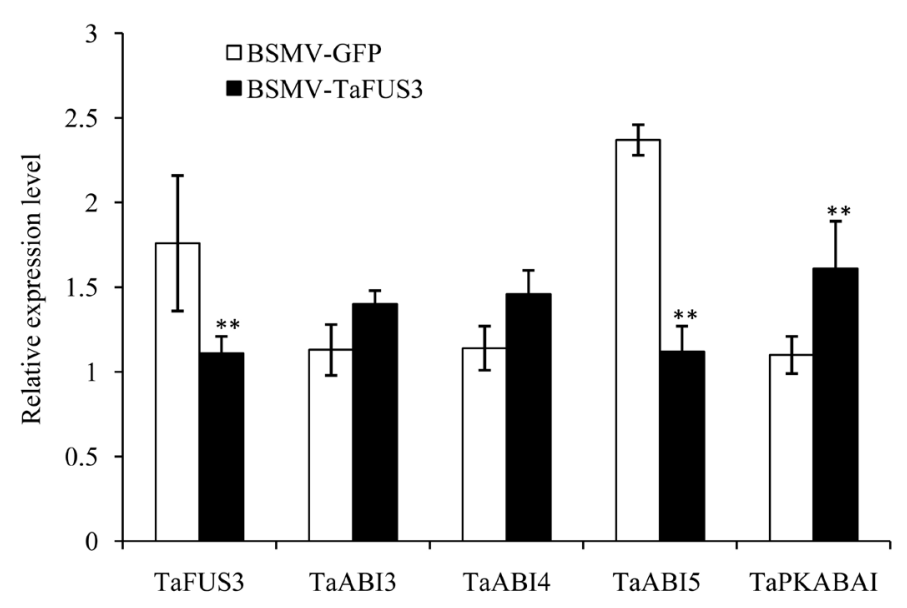

Figure 6. Transcription levels of the TaFUS3, TaABI3, TaABI4, TaPKABA1, and TaABI5 genes in embryos of the BSMV-infected plants after $50 \mu \mathrm{mol} / \mathrm{L}$ ABA treatment. Each value is the mean \pm standard deviation of three independent biological replicates. "**»" indicate significant differences at $p<0.01$ level.

(Figure 6). Although the expression of $A B I 3$ was also up-regulated, the difference was not significant. These findings suggested that repressed expression of TaFUS3 gene reduced ABA sensitivity through negatively regulating $A B I 4$ and $P K A B A 1$ genes and positively regulating $A B I 5$ gene involved in the ABA signaling pathway.

\section{Discussion}

FUS3 is a key regulator in inducing dormancy and inhibiting seed germination in Arabidopsis thaliana. In the present study, in order to clarify the functions of FUS3 orthologs in wheat seed germination and PHS resistance, the cDNA and genomic DNA sequences of TaFUS3 homoeologs from wheat were isolated and characterized for the first time. Sequence analysis revealed that the TaFUS3 homoeologs had the similar structures and numbers of amino acids, and all encode a protein with a conserved B3 domain (Figure 1). Although the three TaFUS3 genes had high homology with barley HVFUS3 (GenBank No.CAL91173) at amino acid level, there were great differences in gene structure among them. Wheat TaFUS3 homoeologs had seven exons and six introns, whereas barley $H_{v F U S 3}$ gene had same six exons and five introns as AtFUS3, implying TaFUS3 gene might have different function from HvFUS3 and AtFUS3.

The qRT-PCR analysis demonstrated that only two copies of the TaFUS3 genes were constitutively transcribed at different developmental and germinating stages. Moreover, transcript levels of both TaFUS3-3B and TaFUS3-3D also varied with the developmental and germinating process. During grain filling stage, the relatively high expression of TaFUS3-3B and TaFUS3-3D was recorded at 25 DAP (Figure 2(a)). Similar results were obtained by Luerßen et al. [17], who showed that the expression level of AtFUS3 was higher in the prophase and near metaphase of seed development, but decreased before seed maturity. The 
two TaFUS3 genes were induced after seed imbibition, and relatively high expression was noted $24 \mathrm{~h}$ after imbibition commenced (Figure 2(b)); these findings were in agreement with the results observed by Chiu et al. at high temperatures [31]. Hence, the expression of the three TaFUS3 genes in wheat seed was differentially regulated, which might reflect a difference in functions of the three homoeologs in the development and germination.

Earlier experiments with the transgenic Arabidopsis revealed that AtFUS3 over-expression delayed seed germination during imbibition [31]. Based on this finding, it might be expected that under-expression of TaFUS3 would have an opposite effect, that is accelerating germination. As shown in Figure 4, this hypothesis was supported; germination of seeds in TaFUS3-silenced plants was hindered for at least $7 \mathrm{~d}$ following the addition of water. Spike sprouting test further revealed that grains in the TaFUS3-silenced plants showed copious germination in the spike $7 \mathrm{~d}$ after being subjected to sprouting conditions, whereas the grain in the plants with normal expression of TaFUS3 barely germinated at 40 dpi. However, germination rate in TaFUS3-silenced plants was significantly lower than those in the control (GFP infected) plants at $45 \mathrm{dpi}$, suggesting the difference narrowed. So, we speculated that this might be related to the decrease in dormancy level of grains because the expression levels in grains of TaFUS3 silenced spikes were lower than those in the control plants. These results suggested that TaFUS3 had an important role in regulating seed dormancy and PHS tolerance. Interestingly, the inhibition of germination occurred at the appropriate seed germination temperature $\left(25^{\circ} \mathrm{C}\right)$ of wheat instead of the supra-optimal temperature in case of Arabidopsis reported by Chiu et al. [31]. The reason might be related to the characteristics of different species.

It has long been known that endogenous hormones abscisic acid (ABA) and gibberellin (GA) played a key role in controlling seed dormancy and germination [12]. GAs breaks seed dormancy and promote germination. However, ABA is considered to induce and maintain seed dormancy. Seed dormancy or germination depends on the levels of ABA/GA, which are controlled by the precise balance between biosynthesis and catabolism rates of these hormones [12]. Recently, the major enzymes involved in ABA and GA metabolism pathways have been identified, such as ABA biosynthetic gene $N C E D$ that encodes 9-cis-epoxycarotenoid dioxygenase, ABA catabolic gene CYP707A that encodes ABA 8'-hydroxylase, GA biosynthetic gene GA20ox that encodes GA 20-oxidase, GA3ox that encodes GA 3-hydroxy-lase, and GA catabolic gene GA2ox that encodes GA 2-oxidase [42] [43] [45]. Gubler et al. [46] reported that suppressing expression of $H_{V} C Y P 707 A$ in transgenic barley grains resulted in a higher ABA content and increased dormancy. The over-expression of AtFUS3 delayed seed germination by regulating ABA/GA ratio at high temperatures [31] [33]. In the present study, seed germination and PHS were significantly enhanced when TaFUS3 gene was down-regulated. Therefore, we hypothesized that the TaFUS3 gene may be involved in the regulation of ABA/GA synthesis and metabolism 
during seed germination. To test the hypothesis, we investigated the expression patterns of the ABA biosynthesis-related genes TaNCED1 and TaNCED2, the GA biosynthesis-related genes TaGA20ox-D4 and TaGA20ox1d and the ABA metabolism-related gene TaCYP707A2. The transcription levels of TaGA20ox-D4, TaGA20ox1d and TaCYP707A2 in grains of BSMV:TaFUS3-silenced plants were significantly up-regulated (Figure 5), whereas the expression levels of TaNCED1 and TaNCED2 in BSMV:TaFUS3-silenced plants were significantly down-regulated in imbibed seed (Figure 5). This pattern is in accordance with the increase in seed germination and PHS capacity observed in BSMV: TaFUS3 silenced plants after $7 \mathrm{~d}$ of incubation (Figure 4). These results suggested that down-regulating expression of TaFUS3 might lead to increased GA transcription level and decreased ABA transcription level, indicating TaFUS3 may regulate seed dormancy and PHS-resistance by regulating the expression levels of genes in the $\mathrm{ABA}$ and GA metabolism pathways.

In addition to $\mathrm{ABA}$ levels, the $\mathrm{ABA}$ signal and sensitivity of embryos to $\mathrm{ABA}$ also play important roles in seed dormancy and PHS. Cultivars with strong dormancy have strong sensitivity to ABA [47] [48] [49]. The ABA signal regulators, such as the $\mathrm{ABI}$ (for $\mathrm{ABA}$-insensitive) transcription factors, include $A B I 3$, $A B I 4$ and $A B I 5$. The $A B I 3$ belongs to the $\mathrm{B} 3$ type transcription factors and plays an important regulatory role in seed dormancy and $A B A$ inhibition of seed germination [50]. The $A B I 5$ encodes a bZIP transcription factor whose accumulation inhibits seed germination and early seedling growth [51] [52]. Another important component of the pathway is the ABA-induced Ser/Thr protein kinase PKABA1 [53]. The PKABA1 mRNA levels in both seeds and leaves of wheat increase rapidly in response to dehydration and ABA [54]. In the present work, we examined the response of TaFUS3 and the ABA signaling pathway-related genes $A B I 3, A B I 4, A B I 5$, and $P K A B A 1$ to ABA treatment. The results revealed that the expression of TaFUS3 and $A B I 5$ was significantly down-regulated and the expression of $A B I 3, A B I 4$ and $P K A B A 1$ was up-regulated in the TaFUS3-silenced plants in the ABA treatment (Figure 6). We speculate that the mechanism underpinning reduced ABA sensitivity of TaFUS3-silenced plants is based on $T a$ FUS3 regulating the expression of genes susceptible as PKABAland ABI5.

\section{Conclusions}

In this study, three TaFUS3 homoeologous genes, encoding B3-domin proteins, were isolated and their role in seed germination and PHS were investigated using the BSMV-VIGS method under field conditions. In TaFUS3-silenced wheat plants, seed germination and PHS capacities were significantly increased, and the seed dormancy level was significantly decreased in mature grains. The transcription levels of two GA synthesis-related genes and one ABA catabolism-related gene were significantly up-regulated, and two ABA synthesis-related genes were significantly down-regulated. In addition, three signal genes involved in ABA response were markedly up-regulated, and one signal gene was markedly down-regulated 
in embryos of TaFUS3-silenced plants under ABA treatment. These results indicated that TaFUS3 acts as a positive regulator in inhibiting seed germination and PHS, and temporally regulates the expression of some ABA, GA metabolic-related genes and $\mathrm{ABA}$ signal genes in imbibed wheat seed.

\section{Acknowledgements}

This work was financially supported by National Key Research and Development Program in China (2017YFD0301101) and Key Scientific and Technological Projects in Henan Province (212102110057).

\section{Conflicts of Interest}

The authors declare no conflicts of interest regarding the publication of this paper.

\section{References}

[1] Bewley, J.D. (1997) Seed Germination and Dormancy. Plant Cell, 9, 1055-1066. https://doi.org/10.1105/tpc.9.7.1055

[2] Gale, M.D. and Lenton, J.R. (1987) Preharvest Sprouting in Wheat: A Complex Genetic and Physiological Problem Affecting Bread Making Quality in UK Wheat. Aspects of Applied Biology, 15, 115-124.

[3] Groos, C., Gay, G., Perretant, M.R., Gervais, L., Bernard, M., Dedryver, F., et al. (2002) Study of the Relationship between Pre-Harvest Sprouting and Grain Color by Quantitative Trait Loci Analysis in a White X Red Grain Bread-Wheat Cross. Theoretical and Applied Genetics, 104, 39-47. https://doi.org/10.1007/s001220200004

[4] Barnard, A. (2001) Genetic Diversity of South African Winter Wheat Cultivars in Relation to Preharvest Sprouting and Falling Number. Euphytica, 119, 109-112. https://doi.org/10.1023/A:1017571212607

[5] Amano, Y. and Torada, A. (2002) Breeding of White-Grained Wheats for Japan. Euphytica, 126, 83-88. https://doi.org/10.1023/A:1019615504609

[6] Kulwal, P., Kumar, N., Gaur, A., Khurana, P., Khurana, J., Tyagi, A., et al. (2005) Mapping of a Major QTL for Pre-Harvest Sprouting Tolerance on Chromosome 3A in Bread Wheat. Theoretical and Applied Genetics, 111, 1052-1059. https://doi.org/10.1007/s00122-005-0021-4

[7] Ogbonnaya, F.C., Imtiaz, M., Ye, G., Hearnden, P.R., Hernandez, E., Eastwood, R.F., et al. (2008) Genetic and QTL Analyses of Seed Dormancy and Preharvest Sprouting Resistance in the Wheat Germplasm CN10955. Theoretical and Applied Genetics, 116, 891-902. https://doi.org/10.1007/s00122-008-0712-8

[8] Xiao, S.H., Zhang, X.Y., Yan, C.S. and Lin, H. (2002) Germplasm Improvement for Preharvest Sprouting Resistance in Chinese White-Grained Wheat: An Overview of the Current Strategy. Euphytica, 126, 35-38. https://doi.org/10.1023/A:1019679924173

[9] Nonogaki, H., Barrero, J.M. and Li, C. (2018) Seed Dormancy, Germination and Pre-Harvest Sprouting. Frontiers in Plant Science, 9, Article No. 1783. https://doi.org/10.3389/fpls.2018.01783

[10] Gubler, F., Millar, A.A. and Jacobsen, J.V. (2005) Dormancy Release, ABA and 
Pre-Harvest Sprouting. Current Opinion in Plant Biology, 8, 183-187. https://doi.org/10.1016/j.pbi.2005.01.011

[11] McCarty, D.R. (1995) Genetic Control and Integration of Maturation and Germination Pathways in Seed Development. Annual Review of Plant Physiology and Plant Molecular Biology, 46, 71-93. https://doi.org/10.1146/annurev.pp.46.060195.000443

[12] Tuan, P.A., Kumar, R., Rehal, P.K., Toora, P.K. and Ayele, B.T. (2018) Molecular Mechanisms Underlying Abscisic Acid/Gibberellin Balance in the Control of Seed Dormancy and Germination in Cereals. Frontiers in Plant Science, 9, Article No. 668. https://doi.org/10.3389/fpls.2018.00668

[13] Finkelstein, R.R., Gampala, S.S.L. and Rock, C.D. (2002) Abscisic Acid Signaling in Seeds and Seedlings. Plant Cell, 14, S15-S45. https://doi.org/10.1105/tpc.010441

[14] Finkelstein, R., Reeves, W., Ariizumi, T. and Steber, C. (2008) Molecular Aspects of Seed Dormancy. Annual Review of Plant Biology, 59, 387-415. https://doi.org/10.1146/annurev.arplant.59.032607.092740

[15] Suzuki, M. and McCarty, D.R. (2008) Functional Symmetry of the B3 Network Controlling Seed Development. Current Opinion in Plant Biology, 11, 548-553. https://doi.org/10.1016/j.pbi.2008.06.015

[16] Giraudat, J., Hauge, B.M., Valon, C., Smalle, J., Parcy, F. and Goodman, H.M. (1992) Isolation of the Arabidopsis ABI3 Gene by Positional Cloning. Plant Cell, 4, 1251-1261. https://doi.org/10.1105/tpc.4.10.1251

[17] Luerßen, H., Kirik, V., Herrmann, P. and Miséra, S. (1998) FUSCA3 encodes a Protein with a Conserved VP1/ABI3-Loke B3 Domain Which Is of Functional Importance for the Regulation of Seed Maturation in Arabidopsis thaliana. Plant Journal, 15, 755-764. https://doi.org/10.1046/j.1365-313X.1998.00259.x

[18] Lotan, T., Ohto, M., Yee, K.M., West, M.A.L., Lo, R., Kwong, R.W., et al. (1998) Arabidopsis LEAFY COTYLEDON1 Is Sufficient to Induce Embryo Development in Vegetative Cells. Cell, 93, 1195-1205. https://doi.org/10.1016/S0092-8674(00)81463-4

[19] Stone, S.L., Kwong, L.W., Yee, K.M., Pelletier, J., Lepiniec, L., Fischer, R.L., et al. (2001) LEAFY COTYLEDONE2 Encodes a B3 Domain Transcription Factor That Induces Embryo Development. Proceedings of the National Academy of Sciences of the United States of America, 98, 11806-11811. https://doi.org/10.1073/pnas.201413498

[20] Jia, H., McCarty, D.R. and Suzuki, M. (2013) Distinct Roles of LAFL Network Genes in Promoting the Embryonic Seedling Fate in the Absence of VAL Repression. Plant Physiology, 163, 1293-1305. https://doi.org/10.1104/pp.113.220988

[21] Nonogaki, H. (2014) Seed Dormancy and Germination-Emerging Mechanisms and New Hypotheses. Frontiers in Plant Science, 5, Article No. 233. https://doi.org/10.3389/fpls.2014.00233

[22] Fatihi, A., Boulard, C., Bouyer, D., Baud, S.S., Dubreucq, B. and Lepiniec, L. (2016) Deciphering and Modifying LAFL Transcriptional Regulatory Network in Seed for Improving Yield and Quality of Storage Compounds. Plant Science, 250, 198-204. https://doi.org/10.1016/j.plantsci.2016.06.013

[23] To, A., Valon, C., Savino, G., Guilleminot, J., Devic, M., Giraudat, J., et al. (2006) A Network of Local and Redundant Gene Regulation Governs Arabidopsis Seed Maturation. Plant Cell, 18, 1642-1651. https://doi.org/10.1105/tpc.105.039925

[24] Sreenivasulu, N. and Wobus, U. (2013) Seed-Development Programs: A Systems Biology-Based Comparison between Dicots and Monocots. Annual Review of Plant 
Biology, 64, 189-217. https://doi.org/10.1146/annurev-arplant-050312-120215

[25] Boulard, C., Fatihi, A., Lepiniec, L. and Dubreucq, B. (2017) Regulation and Evolution of the Interaction of the Seed B3 Transcription Factors with NF-Y Subunits. BBA-Gene Regulatory Mechanisms, 1860, 1069-1078.

https://doi.org/10.1016/j.bbagrm.2017.08.008

[26] Gazzarrini, S., Tsuchiya, Y., Lumba, S., Okamoto, M. and McCourt, P. (2004) The Transcription Factor FUSCA3 Controls Developmental Timing in Arabidopsis through the Hormones Gibberellin and Abscisic Acid. Developmental Cell, 7, 373-385. https://doi.org/10.1016/j.devcel.2004.06.017

[27] Tsai, A.Y.L. and Gazzarrini, S. (2012) AKIN10 and FUSCA3 Interact to Control Lateral Organ Development and Phase Transitions in Arabidopsis. Plant Journal, 69, 809-821. https://doi.org/10.1111/j.1365-313X.2011.04832.x

[28] Carbonero, P., Iglesias-Fernandez, R. and Vicente-Carbajosa, J. (2016) The AFL Subfamily of B3 Transcription Factors: Evolution and Function in Angiosperm Seeds. Journal of Experimental Botany, 68, 871-880. https://doi.org/10.1093/jxb/erw458

[29] Lu, Q.S., Dela-Paz, J., Pathmanathan, A., Chiu, R.S., Tsai, A.Y. and Gazzarrini, S. (2010) The C-Terminal Domain of FUSCA3 Negatively Regulates mRNA and Protein Levels and Mediates Sensitivity to the Hormones Abscisic Acid and Gibberellic acid in Arabidopsis. Plant Journal, 64, 100-113.

https://doi.org/10.1111/j.1365-313X.2010.04307.x

[30] Curaba, J., Moritz, T., Blervaque, R., Parcy, F., Raz, V., Herzog, M., et al. (2004) AtGA3ox2, a Key Gene Responsible for Bioactive Gibberellin Biosynthesis, Is Regulated during Embryogenesis by LEAFY COTYLEDON2 and FUSCA3 in Arabidopsis. Plant Physiology, 136, 3660-3669. https://doi.org/10.1104/pp.104.047266

[31] Chiu, R.S., Nahal, H., Provart, N.J. and Gazzarrini, S. (2012) The Role of the Arabidopsis FUSCA3 Transcription Factor during Inhibition of Seed Germination at High Temperature. BMC Plant Biology, 12, Article No. 15.

https://doi.org/10.1186/1471-2229-12-15

[32] Chiu, R.S., Pan, S., Zhao, R. and Gazzarrini, S. (2016) ABA-Dependent Inhibition of the Ubiquitin Proteasome System during Germination at High Temperature in Arabidopsis. Plant Journal, 88, 749-761. https://doi.org/10.1111/tpj.13293

[33] Chiu, R.S., Saleh, Y. and Gazzarrini, S. (2016) Inhibition of FUSCA3 Degradation at High Temperature Is Dependent on ABA Signaling and Is Regulated by the ABA/GA Ratio. Plant Signaling and Behavior, 11, Article No. e1247137. https://doi.org/10.1080/15592324.2016.1247137

[34] Moreno-Risueno, M.A., Gonzalez, N., Diaz, I., Parcy, F., Carbonero, P. and Vicente-Carbajosa, J. (2008) FUSCA3 from Barley Unveils a Common Transcriptional Regulation of Seed-Specific Genes between Cereals and Arabidopsis. Plant Journal, 53, 882-894. https://doi.org/10.1111/j.1365-313X.2007.03382.x

[35] Sun, F., Liu, X., Wei, Q., Liu, J., Yang, T., Jia, L., et al. (2017) Functional Characterization of TaFUSCA3, a B3-Superfamily Transcription Factor Gene in the Wheat. Frontiers in Plant Science, 8, Article No. 1133. https://doi.org/10.3389/fpls.2017.01133

[36] Liu, G.Y., Wu, Y.F., Xu, M.J., Gao, T., Wang, P.F., Wang, L.N., et al. (2016) Virus-Induced Gene Silencing Identifies an Important Role of the TaRSR1 Transcription Factor in Starch Synthesis in Bread Wheat. International Journal of Molecular Sciences, 17, Article No. 1557. https://doi.org/10.3390/ijms17101557 
[37] Ren, J.P., Wang, X.G. and Meng, X.D. (2019) Wheat Gene TaFUS3 and Its Application. China Patent No. ZL201510970510.6.

[38] Feng, Y.L., Wang, K.T., Ma, C., Zhao, Y.Y. and Yin, J. (2015) Virus-Induced Gene Silencing-Based Functional Verification of Six Genes Associated with Vernalization in Wheat. Biochemical and Biophysical Research Communications, 458, 928-933. https://doi.org/10.1016/j.bbrc.2015.02.064

[39] Pogue, G.P., Lindbo, J.A., Dawson, W.O. and Turpen, T.H. (1998) Tobamovirus Transient Expression Vectors: Tools for Plant Biology and High Level Expression of Foreign Proteins in Plants. In: Gelvin, S.B. and Schilperoot, R.A., Eds., Plant Molecular Biology Manual, Kluwer Academic Publishers, Dordrecht, 67-93. https://doi.org/10.1007/978-94-011-5242-6_5

[40] Wang, X.G., Wang, Y.L., Xiao, R.X., Chen, X. and Ren, J.P. (2016) Molecular Characterization and Expression Analysis of Three Homoeologous Ta14S Genes Encoding 14-3-3 Proteins in Wheat (Triticum aestivum L.). The Crop Journal, 4, 188-198. https://doi.org/10.1016/j.cj.2016.03.002

[41] Livak, K.J. and Schmittgen, T.D. (2001) Analysis of Relative Gene Expression Data Using Real-Time Quantitative PCR and the $2^{-\Delta \Delta C t}$ Method. Methods, 25, 402-408. https://doi.org/10.1006/meth.2001.1262

[42] Seo, M. and Koshiba, T. (2002) Complex Regulation of ABA Biosynthesis in Plants. Trends in Plant Science, 7, 41-48. https://doi.org/10.1016/S1360-1385(01)02187-2

[43] Okamoto, M., Kuwahara, A., Seo, M., Kushiro, T., Asami, T., Hirai, N., et al. (2006) CYP707A1 and CYP707A2, Which Encode Abscisic acid 8'-Hydroxylases, Are Indispensable for Proper Control of Seed Dormancy and Germination in Arabidopsis. Plant Physiology, 141, 97-107. https://doi.org/10.1104/pp.106.079475

[44] Yamauchi, Y., Ogawa, M., Kuwahara, A., Hanada, A., Kamiya, Y. and Yamaguchi, S. (2004) Activation of Gibberellin Biosynthesis and Response Pathways by Low Temperature during Imbibition of Arabidopsis Thaliana Seeds. Plant Cell, 16, 367-378. https://doi.org/10.1105/tpc.018143

[45] Finch-Savage, W.E., Cadman, C.S., Toorop, P.E., Lynn, J.R. and Hilhorst, H.W. (2007) Seed Dormancy Release in Arabidopsis Cvi by dry After-Ripening, Low Temperature, Nitrate and Light Shows Common Quantitative Patterns of Gene Expression Directed by Environmentally Specific Sensing. Plant Journal, 51, 60-78. https://doi.org/10.1111/j.1365-313X.2007.03118.x

[46] Gubler, F., Hughes, T., Waterhouse, P. and Jacobsen, J. (2008) Regulation of Dormancy in Barley by Blue Light and After-Ripening: Effects on Abscisic Acid and Gibberellins Metabolism. Plant Physiology, 147, 886-896.

https://doi.org/10.1104/pp.107.115469

[47] Walker-Simmons, M. (1987) ABA Levels and Sensitivity in Developing Wheat Embryos of Sprouting Resistant and Susceptible Cultivars. Plant Physiology, 84, 61-66. https://doi.org/10.1104/pp.84.1.61

[48] Morris, C.F., Moffatt, J.M., Sears, R.G. and Paulsen, G.M. (1989) Seed Dormancy and Responses of Caryopses, Embryos, and Calli to Abscisic Acid in Wheat. Plant Physiology, 90, 643-647. https://doi.org/10.1104/pp.90.2.643

[49] Corbineau, F., Abdelilah, B. and Côme, D. (2000) Changes in Sensitivity to Abscisic Acid of the Developing and Maturing Embryo of Two Wheat Cultivars with Different Sprouting Susceptibility. Israel Journal of Plant Sciences, 48, 189-197.

[50] Yang, Y., Ma, Y.Z., Xu, Z.S., Chen, X.M., He, Z.H., Wilkinson, M., et al. (2007) Isolation and Characterization of Viviparous 1 Genes in Wheat Cultivars with Distinct 
ABA Sensitivity and Pre-Harvest Sprouting Tolerance. Journal of Experimental Botany, 58, 2863-2871. https://doi.org/10.1093/jxb/erm073

[51] Finkelstein, R.R. and Lynch, T.J. (2000) The Arabidopsis Abscisic Acid Response Gene $A B I 5$ Encodes a Basic Leucine Zipper Transcription Factor. Plant Cell, 12, 599-609. https://doi.org/10.1105/tpc.12.4.599

[52] Lopez-Molina, L., Mongrand, S. and Chua, N.H. (2001) A Post Germination Developmental Arrest Checkpoint Is Mediated by Abscisic Acid and Requires the ABI5 Transcription Factor in Arabidopsis. Proceedings of the National Academy of Sciences of the United States of America, 98, 4782-4787. https://doi.org/10.1073/pnas.081594298

[53] Anderberg, R.J. and Walker-Simmons, D.M.K. (1992) Isolation of a Wheat cDNA Clone for an Abscisic Acid-Inducible Transcript with Homology to Protein Kinases. Proceedings of the National Academy of Sciences of the United States of America, 89, 10183-10187. https://doi.org/10.1073/pnas.89.21.10183

[54] Holappa, L.D. and Walker-Simmons, D.M.K. (1995) The Wheat Abscisic Acid-Responsive Protein Kinase mRNA, PKABA1, Is Up-Regulated by Dehydration, Cold Temperature, and Osmotic Stress. Plant Physiology, 108, 1203-1210.

https://doi.org/10.1104/pp.108.3.1203 